\title{
Challenges, reorganisation and recovery of obstetrics and gynecology outpatient services during and after the COVID-19 pandemic in a tertiary hospital
}

\author{
Meei Jiun Seet ${ }^{1}$, Krystal Koh $^{1}$, Manisha Mathur ${ }^{2}$ \\ ${ }^{1}$ Department of Obstetrics and Gynecology, KK Women's and Children's Hospital, Singapore; 'Department of Obstetrics and Gynecology, KK \\ Women's and Children's Hospital, Yong Loo Lin School of Medicine, Duke NUS Medical School, Lee Kong Chian School of Medicine, Singapore \\ Contributions: (I) Conception and design: MJ Seet; (II) Administrative support: All authors; (III) Provision of study materials or patients: None; (IV) \\ Collection and assembly of data: None; (V) Data analysis and interpretation: None; (VI) Manuscript writing: All authors; (VII) Final approval of \\ manuscript: All authors. \\ Correspondence to: Krystal Koh. Resident, Department of Obstetrics and Gynecology, KK Women's and Children's Hospital, 100 Bukit Timah Road, \\ Singapore 229899, Singapore. Email: krystalgermaine@gmail.com.
}

\begin{abstract}
The coronavirus disease 2019 (COVID-19) infection was declared a pandemic by the World Health Organization (WHO) on 11 March 2020. As of 16 January 2021, the WHO reported a total of $92,506,811$ cases globally, with 2,001,773 deaths. The total number of confirmed COVID-19 infections in Singapore reported by the Ministry of Health $(\mathrm{MOH})$ as of 16 January 2021 is 59,054 cases with 270 active cases. As there is currently no cure for COVID-19 infection, the best management strategy remains prevention of transmission through public infection control measures and the recently introduced vaccination programs. Globally, healthcare institutions across multiple disciplines are actively taking measures to reduce their ambulatory outpatient load for low-risk conditions to avoid unnecessary clinic visits and hence reduce the risk of exposure and transmission. In this paper, we describe the challenges faced and the reorganisation of care in the outpatient Obstetrics and Gynaecology (O\&G) service in the largest tertiary hospital and referral centre for O\&G in Singapore during the COVID-19 outbreak. We also present the challenges we anticipated upon resumption of our services in the post-outbreak period, and discuss the novel strategies we implemented to cope with the increased number of cases expected. These strategies are aimed to improve the standard of patient care, whilst adhering to public infection control guidelines to ensure the safety of our patients and healthcare workers.
\end{abstract}

Keywords: Coronavirus disease 2019 (COVID-19); ambulatory care; infection control; obstetrics; gynaecology

Received: 26 November 2020; Accepted: 28 February 2021; Published: 25 June 2021.

doi: $10.21037 /$ jhmhp-20-150

View this article at: http://dx.doi.org/10.21037/jhmhp-20-150

\section{Introduction}

The coronavirus disease 2019 (COVID-19) infection was declared a pandemic by the World Health Organization (WHO) on 11 March 2020 (1). As of 16 January 2021, the WHO reported a total of $92,506,811$ cases globally, with 2,001,773 deaths (2).

The number of confirmed COVID-19 infections in Singapore reported by the Ministry of Health (MOH) as of 16 January 2021 is 59,054 cases with 270 active cases (3).
As there are currently no curative treatment options for COVID-19, the best management strategy is prevention of transmission through infection control measures, and the more recently introduced vaccination programs.

In the outpatient setting, healthcare institutions globally have restructured the ambulatory service to incorporate telemedicine in providing care to low-risk patients to minimise the risk of exposure during the pandemic $(4,5)$. Stable benign gynaecological conditions such as fibroids, 
in the absence of uncontrollable symptoms or risk factors for malignancy, are suitable for tele-consultation. On the other hand, conditions where malignancy cannot be excluded, such as post-menopausal bleeding, or suspicious uterine or adnexal masses, require urgent face-to-face assessment. Gynae-oncology, obstetrics and abortion services are essential and time sensitive. Gynae-oncology units have thus continued their services with modifications to triage the most urgent cases that need to be reviewed in clinic, or undergo surgical, chemotherapy or radiotherapy treatments $(6,7)$.

Globally, healthcare institutions across multiple disciplines are actively taking measure to reduce their ambulatory outpatient load for low-risk conditions (814). In this paper, we describe the challenges faced and the reorganisation of care in the outpatient obstetric and gynaecology $(\mathrm{O} \& \mathrm{G})$ service in our tertiary institution.

\section{Challenges and reorganisation of outpatient service in our tertiary institution}

The outpatient services for subsidised patients in our institution is mainly provided by doctors and supported by midwives and Advanced Practice Nurses (APN). We run services such as antenatal and postnatal care, general and complex gynaecology, abortion and contraception, as well as providing these services in our partner institution and the prison.

\section{Increase in obstetric workload}

With the escalation of Disease Outbreak Response System Condition (DORSCON) from Yellow to Orange on the $7^{\text {th }}$ of February 2020, the number of new Obstetrics referral has increased from an average of 340 per month to more than 600 per month from February 2020 and peaked at 696 cases in April 2020. The reason for this increase is likely due to the cessation of our partner institution and prison services with diversion of their patients to our unit. In addition, the two other restructured obstetric units in Singapore had limited the number of new referrals to cope with the redistribution of manpower to other departments. We conducted two extra obstetric clinics per day to cope with the increased workload.

\section{Increase in abortion workload}

Our abortion service saw more than 50 percent increase in the number of women requesting for termination of pregnancy (TOP). As TOP is time-sensitive, we added three extra clinics per month in May 2020. Patients who have completed their abortion were followed up with teleconsultation by experienced nurses as most do not require a physical examination.

\section{Reorganisation of gynaecology services}

The overall number of general gynaecology patients seen in our institution was reduced during this period, as our national primary care services had been advised to only make urgent referrals during this period based on a provided referral guide, and new referrals were additionally vetted by consultants for further triaging. Follow-up patients were triaged with postponement of non-urgent cases and in April 2020, during the Circuit Breaker (CB) period, these patients were contacted by doctors via telephone call for triaging and consultation. Repeat prescriptions were sent to patients via a complimentary courier service. Instead of 12 general gynaecology clinics per day before the pandemic, we conducted only four clinics per day during the CB.

Women seeking contraceptive implants were at risk of getting unplanned pregnancies. In Singapore, besides male condoms, women can only obtain contraception from either a general practitioner (GP) or a gynaecologist. We have thus maintained the number of contraceptive implant clinics at two per week, with tele-consultation to follow-up these patients. On the other hand, the Well Women Clinic run by the APN was postponed during this period, as the patients did not require urgent review. Moreover, the services of the APN were required at the inpatient ward level due to nursing manpower crisis.

\section{Safety measures to reduce risk of exposure to COVID-19}

During the COVID-19 outbreak, in line with global and national infection control policies, our institution adopted a strict triaging criterion for patients seen at the outpatient clinics, with mandatory health and travel declarations and temperature screening. There was a dedicated room and doctor assigned to see cases with respiratory symptoms, fever, or positive travel and/or contact history, which were deemed urgent to have a face-to-face consultation on that day, and not suitable for postponement. The assigned doctor would don full personal protective equipment (PPE) during the consultation and the room fully disinfected afterwards. 


\section{Challenges during recovery period}

As we resumed our services after withdrawal of $\mathrm{CB}$, we anticipated significant challenges, including the increased obstetrics and abortion workload and coping with the backlog of postponed gynaecology patients. At the same time, all measures such as 'safe distancing' and screening for temperature and travel history continued to be essential.

Obstetrics patients have more frequent antenatal visits in their third trimester. We foresee the need for more obstetrics clinics at the end of 2020 to cope with this increased demand. We also predict a surge in the number of women seeking abortion in the next few months based on recent trends. As for general gynaecology, the backlog of returning patients and the continued discontinuation of our partner institution and prison services will continue to impact our outpatient workload. We plan to continue triaging new referrals from primary care to allow time to clear the backlog of cases.

\section{Discussion}

The COVID-19 outbreak has resulted in major changes to our subsidised outpatient $O \& G$ service. Though challenging, this period has provided us the opportunity to review and rectify deficiencies within our system. With a strong team, consisting of doctors, nurses, clinical operations and manpower teams, we managed to abide by safe distancing measures, prevent overcrowding whilst providing safe medical care. As we enter a different phase after the $\mathrm{CB}$, our team has implemented innovative improvements on how our outpatient clinics are run, to provide better patient care.

\section{Adoption of telemedicine}

The quote "Necessity is the mother of all inventions" from Greek philosopher Plato's 'Republic' seems applicable in the current circumstances and can be modified to add that in order to survive we must learn to be flexible and adapt constantly. During these times, the healthcare services in Singapore and elsewhere have undergone significant adaptations. In line with infection control measures, and in tandem with institutions globally, we have adopted telemedicine to reduce the number of non-urgent face-toface consultations. Prior to the outbreak, telemedicine was not offered in the $\mathrm{O} \& \mathrm{G}$ outpatient services. Hence, while newly incorporating telemedicine, we did not have any standard protocol for such consultations. Over a short span of time and experience, we developed an evolving structure for verifying the patients' identities and documenting in a consistent format within the electronic medical records (EMR).

As this is a new service and so far not reported widely in $\mathrm{O} \& \mathrm{G}$, we conducted a survey on 88 follow-up patients with general gynaecological conditions, who had been tele-consulted during the $\mathrm{CB}$ and 63 (71.6\%) of them felt that the doctor was able to meet their medical needs and provide adequate information about their condition via tele-consultation. However, only 51 (58.0\%) felt that telemedicine should continue to replace some of the faceto-face consultations in $\mathrm{O} \& \mathrm{G}$.

As our outpatient services resume, we identified a subgroup of patients who are suitable for tele-consultation, including follow-up patients after abortion and contraception. Women are assessed for their suitability for tele-consultation during their first face-to-face consultation and written consent is obtained at the same setting. With a more streamlined process in place, we hope our healthcare professionals and patients will be able to get better experience with telemedicine. We plan to carry out more studies on patient satisfaction and outcomes from teleconsultations, with a view to improve and expand the use of telemedicine in our services.

However, we do acknowledge drawbacks of adopting telemedicine, including limitations to access for patients of lower socioeconomic or educational status, patients who are socially or medically isolated, and patients who are victims of domestic violence. Bradley et al. had described the challenges of telemedicine for victims of intimate partner violence during the COVID pandemic as tele-consultation with such patients who are isolated at home may occur in an environment where the perpetrator of violence is also present (15). Technological challenges such as connectivity issues or hardware malfunction may also compromise teleconsultations. An additional but important drawback of telemedicine includes the inability to properly assess the body language or facial expressions of the patient, which may impact on the patient-doctor relationship and care of the patient.

\section{Training and involvement of non-medical healthcare providers}

To cope with the anticipated increased number of patients post-CB, we have trained and designed greater roles for 
the APNs, midwives and staff nurses with special interest to conduct low-risk clinics to assist with the workload, thus allowing the doctors to focus on more complex patients. As a long-term plan, these group of nurses and midwives will be able to work along with the doctors to provide more holistic care within our institution.

\section{Collaboration with primary healthcare services}

We will also continue to collaborate with our national primary healthcare services to utilise their capacity for managing low-risk gynaecological conditions, and hence reduce the burden on tertiary gynaecological services. This will include developing guidelines for the management of common gynaecological problems and providing training for GPs. One area of potential development is training and instituting facilities for low-risk antenatal care to be conducted at the community level, which is currently not offered in the Singapore healthcare system.

\section{Better contraception education and services}

To reduce the number of women seeking abortion, contraceptive education is essential. This requires support at the national level to increase public education through media, schools and primary healthcare centres. Training of GPs in providing long-acting reversible contraception (LARC), such as intrauterine contraceptive devices (IUCDs) or contraceptive implants, can reduce the barrier that some women seeking contraception may face, such as cost or inconvenience of getting referral to a tertiary centre. Our department plans to run more contraception courses for GPs and offer talks for patients and at schools to improve awareness.

\section{Upgrading of facilities}

The increased number of obstetrics patients and women seeking abortion during this period caused a strain in our institution's ultrasound scan department, causing long waiting times. We worked with our hospital management team to purchase extra ultrasound scan machines to conduct basic scans in the clinic, to take the load off our scanning department and reduce clinic waiting time. This also creates more learning opportunities for our resident doctors. We have also reviewed and improved manpower allocation within the clinics with the consideration of running evening and weekend clinics as a temporizing measure to clear the backlog of patients.

\section{Welfare of our clinical staff}

During this pandemic crisis period, we have learnt to be cognizant of the mental health of our staff to avoid burn out. In an unpublished study on resilience in 46 doctors and nurses during this pandemic period using the Brief Resilience Scale, the mean resilience score was 3.7 (normal resilience). We also found that male staff reported significantly higher resilience score as compared to female staff, and doctors score higher than nurses. This result is shared with the ground staff to create awareness and measures such as self-help portals, support and various interactive sessions between the senior and junior staff were put in place constantly for improving resilience and early recognition of mental health issues, and this will be continued even after this pandemic period.

Training needs for our residents were also constantly being reviewed. The national residency protected time teaching sessions were delivered through video conferencing. Interestingly, by doing so, the attendance rate to these teaching sessions has increased significantly from $80 \%$ to more than $95 \%$ during this period. In the future, as we recover from the pandemic, when physical teaching sessions resume, we may consider including video conferencing to allow residents to learn remotely.

\section{Conclusions}

The challenges we faced both during the COVID-19 outbreak and the following recovery period, have given us an opportunity to restructure our outpatient service for the betterment of our services and patients alike. This includes innovating, adopting and developing new services such as telemedicine. We also learn the need to plan ahead and the importance of close collaboration at the national healthcare level with greater emphasis on education and training for GPs, to improve our healthcare system as a whole.

\section{Acknowledgments}

Funding: None.

\section{Footnote}

Conflicts of Interest: All authors have completed the ICMJE uniform disclosure form (available at http://dx.doi. 
org/10.21037/jhmhp-20-150). The authors have no conflicts of interest to declare.

Ethical statement: The authors are accountable for all aspects of the work in ensuring that questions related to the accuracy or integrity of any part of the work are appropriately investigated and resolved.

Open Access Statement: This is an Open Access article distributed in accordance with the Creative Commons Attribution-NonCommercial-NoDerivs 4.0 International License (CC BY-NC-ND 4.0), which permits the noncommercial replication and distribution of the article with the strict proviso that no changes or edits are made and the original work is properly cited (including links to both the formal publication through the relevant DOI and the license). See: https://creativecommons.org/licenses/by-nc-nd/4.0/.

\section{References}

1. World Health Organization (WHO) Emergencies Press Conference on coronavirus disease outbreak - 11 March 2020. World Health Organization (WHO); 2020 [cited 2020 Jun 6]. Available online: https://www.who.int/ emergencies/diseases/novel-coronavirus-2019/mediaresources/press-briefings

2. World Health Organization (WHO) Coronavirus disease (COVID-19) Situation Report - 137. World Health Organization (WHO); 2020 [cited 2020 Jun 6]. Available online: https://www.who.int/emergencies/diseases/novelcoronavirus-2019/situation-reports

3. MOH Updates on COVID-19 (Coronavirus Disease 2019) Local Situation. Ministry of Health (MOH) Singapore; 2020 [cited 2020 Jun 6]. Available online: https://www. moh.gov.sg/covid-19

4. Rimmer M, Al Wattar B, UKARCOG Members, et al. Provision of obstetrics and gynaecology services during the COVID-19 pandemic: a survey of junior doctors in the UK National Health Service. BJOG 2020;127:1123-8.

5. Ahmed S, Sanghvi K, Yeo D. Telemedicine takes centre stage during COVID-19 pandemic. BMJ Innov 2020;6:252-4.

6. Ramirez PT, Chiva L, Eriksson AGZ, et al. COVID-19 Global Pandemic: Options for Management of Gynecologic Cancers. Int J Gynecol Cancer
2020;30:561-3.

7. Lim YH, Chay WY, Wong WL. Surviving the COVID-19 pandemic: early response of a gynecologic oncology unit in Singapore. J Gynecol Oncol 2020;31:e77.

8. Dotters-Katz SK, Hughes BL. Considerations for Obstetric Care during the COVID-19 Pandemic. Am J Perinatol 2020;37:773-9.

9. Chen Y, Li Z, Zhang Y, et al. Maternal health care management during the outbreak of coronavirus disease 2019. J Med Virol 2020;17:jmv.25787.

10. Coronavirus (COVID-19) Infection in Pregnancy. Royal College of Obstetricians \& Gynaecologists (RCOG); 2020.

11. Poon LC, Yang H, Dumont S, et al. ISUOG Interim Guidance on coronavirus disease 2019 (COVID-19) during pregnancy and puerperium: information for healthcare professionals - an update. Ultrasound Obstet Gynecol 2020;55:848-62.

12. Joint RCOG, BSGE and BGCS guidance for the management of abnormal uterine bleeding in the evolving Coronavirus (COVID-19) pandemic. Royal College of Obstetricians \& Gynaecologists (RCOG), British Society for Gynaecological Endoscopy (BSGE) and British Gynaecological Cancer Society (BGCS); 2020.

13. Royal College of Obstetricians \& Gynaecologists (RCOG). Coronavirus (COVID-19) infection and abortion care. 2020. Available online: https://www.rcog.org.uk/en/ guidelines-research-services/guidelines/coronavirusabortion/

14. Advice for women seeking contraception, abortion and other sexual and reproductive healthcare during the COVID-19 pandemic. The Faculty of Sexual \& Reproductive Healthcare (FSRH); 2020 [cited 2020 Jun 6]. Available online: https://www.fsrh.org/documents/advicefor-women-seeking-contraception-abortion-and-other/

15. Bradley NL, DiPasquale AM, Dillabough K, et al. Health care practitioners' responsibility to address intimate partner violence related to the COVID-19 pandemic. CMAJ 2020;192:E609-10.

doi: $10.21037 /$ jhmhp-20-150

Cite this article as: Seet MJ, Koh K, Mathur M. Challenges, reorganisation and recovery of obstetrics and gynecology outpatient services during and after the COVID-19 pandemic in a tertiary hospital. J Hosp Manag Health Policy 2021;5:19. 